\title{
A MODEL OF A RADIALLY SYMMETRIC CLOUD OF SELF-ATTRACTING PARTICLES
}

Abstract. We consider a parabolic equation which describes the gravitational interaction of particles. Existence of solutions and their convergence to stationary states are studied.

Introduction. Consider a cloud of particles in $\mathbb{R}^{n}$ mutually interacting via the gravitational potential generated by themselves, and suppose that the particles are subject to a frictional, velocity-dependent force and a random fluctuation. We are interested in time evolution of the mass distribution in the cloud.

The evolution of the density $f(x, v, t)$ of this system in the phase space $(x, v)$ is described by the equation of Vlasov-Fokker-Planck type [10]

$$
f_{t}=-\nabla_{x} \cdot(v f)+\nabla_{v} \cdot\left(\left(\nabla_{x} \Phi+\beta v\right) f\right)+\beta \Delta_{v} f,
$$

where $\Phi$ is the gravitational potential of the cloud. For the sake of simplicity we put all physical constants (except for $\beta>0$ ) equal to one.

We will consider only the case when the $x$-space is at most 3 -dimensional. Then the gravitational potential $\Phi$ is equal to

$$
\Phi(x, t)=\int_{\mathbb{R}^{n}} \varrho(y, t) E_{n}(x-y) d y,
$$

where

$$
\varrho(x, t)=\int_{\mathbb{R}^{n}} f(x, v, t) d v
$$

is the spatial density of particles and $E_{n}(s)$ is the fundamental solution of the Laplacian in $\mathbb{R}^{n}$, i.e. $E_{1}(s)=|s| / 2, E_{2}(s)=(2 \pi)^{-1} \ln |s|, E_{3}(s)=$

1991 Mathematics Subject Classification: 35K60, 35B40.

Key words and phrases: nonlinear parabolic equation, radially symmetric solutions, asymptotic behavior, cloud of particles.

The preparation of this paper was supported by the KBN grant 0472/P3/94/07. 
$-|s|^{-1} /(4 \pi)$ for $n=1,2,3$ respectively.

We expect (for details see [10]) that in the adiabatic limit of large friction $(\beta \rightarrow \infty)$ the density $\varrho$, which we are interested in, satisfies the FokkerPlanck equation

$$
\varrho_{t}=\nabla \cdot(\nabla \varrho+\varrho \nabla \Phi) .
$$

Assume that $\varrho$ is radially symmetric and define the integrated density $Q(r, t)=\int_{B_{r}} \varrho(x, t) d x$. This satisfies the parabolic equation

$$
Q_{t}=Q_{r r}-(n-1) r^{-1} Q_{r}+\sigma_{n}^{-1} r^{1-n} Q Q_{r},
$$

where $\sigma_{n}$ is the area of the unit sphere in $\mathbb{R}^{n}$.

The equation (4) is supplemented with the initial and boundary conditions

$$
\begin{gathered}
Q(r, 0)=Q_{0}(r) \quad \text { for } r \geq 0, \\
Q(0, t)=0, \quad Q(+\infty, t)=M \quad \text { for } t \geq 0 .
\end{gathered}
$$

The parameter $M, 0 \leq M \leq+\infty$, can be interpreted as the total mass of particles.

In [2], [3], [4], the equation (3) was considered in a bounded domain with a natural no-flux boundary condition. In these papers the questions of existence of solutions, their uniqueness, regularity, and convergence to steady states as time tends to infinity were studied. Moreover, for large mass $M$ the nonexistence of stationary solutions ([4], [8], [9]) and blow-up phenomena for the evolution problem were shown ([2], [4]).

For a discussion of self-similar solutions of (3) in $\mathbb{R}^{n}, n \geq 2$, we refer to $[1]$.

In Section 1 the existence, uniqueness and asymptotic behaviour of solutions of the problem (4)-(6) in the one-dimensional case are considered. Section 2 deals with stationary solutions of our problem in $\mathbb{R}^{n}, n=2,3$, and nonexistence of global-in-time solutions of the nonstationary problem in $\mathbb{R}^{2}$.

1. For $n=1$ the equation (4) takes the form

$$
Q_{t}=Q_{r r}+Q Q_{r}
$$

known as Burgers' equation [7].

Define $\mathbb{R}_{+}^{2}=\{(r, t): r, t>0\}, \mathbb{R}_{+}=[0,+\infty)$.

We say that $Q \in C^{2}\left(\mathbb{R}_{+}^{2}\right) \cap C^{1}\left(\overline{\mathbb{R}_{+}^{2}}\right)$ is a classical solution of the problem (4)-(6) if $Q$ satisfies (4) in $\mathbb{R}_{+}^{2}$ and the conditions (5), (6).

Throughout this section we assume that the initial data $Q_{0}(r)$ is a continuous nondecreasing function such that $Q_{0}(0)=0$ and $Q_{0}(+\infty)=M<+\infty$.

THEOREM 1. There exists a unique classical solution $Q(r, t)$ of the problem (5)-(7). 
Proof. Let us introduce a new dependent variable $v$ by the formula $Q=2 v_{r} / v$, which represents the Hopf-Cole transformation [7]. It is easy to check that $Q$ is a solution of (5)-(7) if and only if $v$ is a positive solution of the problem

$$
\begin{aligned}
v_{t} & =v_{r r}, \\
v(r, 0) & =\exp \left(\frac{1}{2} \int_{0}^{r} Q_{0}(s) d s\right) \equiv f(r), \\
v_{r}(0, t) & =0 .
\end{aligned}
$$

It is well known $([6, \mathrm{Ch} .1])$ that $(8)-(10)$ has a unique positive solution $v$ and

$$
\begin{aligned}
v(r, t) & =\frac{1}{\sqrt{4 \pi t}} \int_{0}^{+\infty}(K(r-\xi, t)+K(r+\xi, t)) f(\xi) d \xi \\
v_{r}(r, t) & =\frac{1}{2 \sqrt{4 \pi t}} \int_{0}^{+\infty}(K(r-\xi, t)-K(r+\xi, t)) f(\xi) Q_{0}(\xi) d \xi
\end{aligned}
$$

where $K(s, t)=\exp \left(-s^{2} /(4 t)\right)$.

Combining (11), (12) and the Hopf-Cole transformation we get

$$
Q(r, t)=\frac{\int_{0}^{+\infty}(K(r-\xi, t)-K(r+\xi, t)) f(\xi) Q_{0}(\xi) d \xi}{\int_{0}^{+\infty}(K(r-\xi, t)+K(r+\xi, t)) f(\xi) d \xi}
$$

From (13) we see that $0 \leq Q(r, t) \leq M$ for $t>0, r>0$.

For a fixed $\varepsilon>0$ we choose $N$ such that $M-Q_{0}(\xi)<\varepsilon$ for $\xi>N$. For such $N$ we can write the following inequality:

$$
\begin{aligned}
Q(r, t) \geq & (M-\varepsilon) \frac{\int_{0}^{+\infty}(K(r-\xi, t)-K(r+\xi, t)) f(\xi) d \xi}{\int_{0}^{+\infty}(K(r-\xi, t)+K(r+\xi, t)) f(\xi) d \xi} \\
& -(M-\varepsilon) \frac{\int_{0}^{N}(K(r-\xi, t)-K(r+\xi, t)) f(\xi) d \xi}{\int_{0}^{+\infty}(K(r-\xi, t)+K(r+\xi, t)) f(\xi) d \xi}
\end{aligned}
$$

valid for $r>0, t>0$. The first term on the right hand side of the last formula tends to $M-\varepsilon$ as $r \rightarrow+\infty$, the second one goes to 0 . Hence $Q(r, t)$ converges to $M$, and it is easy to see that the convergence is locally uniform in $t$.

Remark 1 . Let $Q$ and $\widetilde{Q}$ be the solutions of $(5)-(7)$ starting from the initial data $Q_{0}, \widetilde{Q}_{0}$. Using $(13)$ it can be proved in a standard way that for each $T>0$ and $\varepsilon>0$ there exists $\delta>0$ such that $\left|Q_{0}(r)-\widetilde{Q}_{0}(r)\right|<\delta$ for $r \geq 0$ implies $|Q(r, t)-\widetilde{Q}(r, t)|<\varepsilon$ for $0<t<T$ and $r \geq 0$. 
THEOREM 2. If $t$ tends to $+\infty$, then the solution of (5)-(7) converges to the stationary solution $Q^{s}(r)=M\left(e^{M r}-1\right) /\left(e^{M r}+1\right)=M \tanh (M r / 2)$ locally uniformly in $r$.

Proof. A stationary solution $Q^{s}(r)$ of (5)-(7) satisfies

$$
\begin{gathered}
Q_{r r}^{s}+Q^{s} Q_{r}^{s}=\left(Q_{r}^{s}+\left(Q^{s}\right)^{2} / 2\right)_{r}=0, \\
Q^{s}(0)=0, \quad Q^{s}(+\infty)=M .
\end{gathered}
$$

Clearly, this problem is integrable and its unique solution is

$$
Q^{s}(r)=M \frac{e^{M r}-1}{e^{M r}+1}=M \tanh (M r / 2) .
$$

Define

$$
\begin{aligned}
\Phi_{1}(r, t)= & \int_{-\infty}^{r / \sqrt{4 t}} \exp \left(-y^{2}-M \sqrt{t} y+\frac{1}{2} \int_{0}^{r-\sqrt{4 t} y} \varphi(s) d s\right) d y, \\
\Phi_{2}(r, t)= & \int_{r / \sqrt{4 t}}^{+\infty} \exp \left(-y^{2}+M \sqrt{t} y+\frac{1}{2} \int_{0}^{\sqrt{4 t} y-r} \varphi(s) d s\right) d y \\
\Phi_{3}(r, t)= & \int_{-\infty}^{r / \sqrt{4 t}} \exp \left(-y^{2}-M \sqrt{t} y+\frac{1}{2} \int_{0}^{r-\sqrt{4 t} y} \varphi(s) d s\right) \\
& \times \varphi(r-\sqrt{4 t} y) d y, \\
\Phi_{4}(r, t)= & \int_{r / \sqrt{4 t}}^{+\infty} \exp \left(-y^{2}+M \sqrt{t} y+\frac{1}{2} \int_{0}^{\sqrt{4 t} y-r} \varphi(s) d s\right) \\
& \times \varphi(\sqrt{4 t} y-r) d y,
\end{aligned}
$$

where $\varphi(r)=Q_{0}(r)-M$. It follows from our assumption imposed on $Q_{0}$ that $-M \leq \varphi(r) \leq 0$ and $\varphi(r) \rightarrow 0$ as $r \rightarrow+\infty$. Introducing the new variable $y=(r-\xi) / \sqrt{4 t}$ we get

$$
\int_{0}^{+\infty} K(r-\xi, t) f(\xi) d \xi=\sqrt{4 t} \exp (M r / 2) \Phi_{1}(r, t) .
$$

In a similar way, using the new variable $y=(r+\xi) / \sqrt{4 t}$ we have

$$
\int_{0}^{+\infty} K(r+\xi, t) f(\xi) d \xi=\sqrt{4 t} \exp (-M r / 2) \Phi_{2}(r, t) .
$$

Similarly we can transform the integrals in (12), and in this way we get

$$
Q(r, t)=\frac{e^{M r / 2}\left(M \Phi_{1}(r, t)+\Phi_{3}(r, t)\right)-e^{-M r / 2}\left(M \Phi_{2}(r, t)+\Phi_{4}(r, t)\right)}{e^{M r / 2} \Phi_{1}(r, t)+e^{-M r / 2} \Phi_{2}(r, t)} .
$$


To prove the theorem it is enough to show that $\Phi_{1} / \Phi_{2}$ tends to 1 , and $\Phi_{3} / \Phi_{2}$, $\Phi_{4} / \Phi_{2}$ converge to 0 as $t \rightarrow+\infty$.

Putting

$$
\begin{aligned}
& I_{1}(r, t)=\int_{r / \sqrt{4 t}}^{+\infty} \exp \left(-y^{2}+M \sqrt{t} y+\frac{1}{2} \int_{0}^{\sqrt{4 t} y+r} \varphi(s) d s\right) d y \\
& I_{2}(r, t)=\int_{-r / \sqrt{4 t}}^{r / \sqrt{4 t}} \exp \left(-y^{2}+M \sqrt{t} y+\frac{1}{2} \int_{0}^{r+\sqrt{4 t} y} \varphi(s) d s\right) d y
\end{aligned}
$$

we have $\Phi_{1}(r, t)=I_{1}(r, t)+I_{2}(r, t)$. Now we fix $r$ and choose $N>r$ such that $|\varphi(s)|<\varepsilon$ for $s>N-r$. For such $N$ we get the following inequality:

$$
\begin{aligned}
I_{1}(r, t) \geq & \int_{N / \sqrt{4 t}}^{+\infty} \exp \left(-y^{2}+M \sqrt{t} y+\frac{1}{2} \int_{0}^{\sqrt{4 t} y-r} \varphi(s) d s\right. \\
& \left.+\frac{1}{2} \int_{\sqrt{4 t} y-r}^{\sqrt{4 t} y+r} \varphi(s) d s\right) d y \\
\geq & \exp (-\varepsilon r)\left(\Phi_{2}(r, t)-\int_{r / \sqrt{4 t}}^{N / \sqrt{4 t}} \exp \left(-y^{2}+M \sqrt{t} y\right.\right. \\
& \left.\left.+\frac{1}{2} \int_{0}^{\sqrt{4 t} y-r} \varphi(s) d s\right) d y\right) \\
\geq & \exp (-\varepsilon r)\left(\Phi_{2}(r, t)-\frac{N-r}{\sqrt{4 t}} \exp \left(-\frac{r^{2}}{4 t}+\frac{M N}{2}\right)\right),
\end{aligned}
$$

valid for $r>0, t>0$. Recalling that $\varphi(s) \geq-M$, we have $\Phi_{2}(r, t) \geq$ $\sqrt{\pi} e^{M r / 2} / 4$, for sufficiently large $t$. Combining (17) and the above estimate we obtain

$$
\exp (-\varepsilon r)(1-\varepsilon) \leq \frac{I_{1}(r, t)}{\Phi_{2}(r, t)} \leq 1,
$$

valid for large $t$ and small $\varepsilon$. Obviously $I_{2} / \Phi_{2} \rightarrow 0$ as $t \rightarrow+\infty$. Hence (18) shows that $\Phi_{1} / \Phi_{2} \rightarrow 1$. Similarly we can check that $\Phi_{3} / \Phi_{2}$ and $\Phi_{4} / \Phi_{2}$ tend to 0 . This completes the proof of Theorem 2 .

From Theorem 2 and Remark 1 we deduce the following

Remark 2. For fixed $N>0, \varepsilon>0$ and initial data $Q_{0}, \widetilde{Q}_{0}$ there exists $\delta>0$ such $\left|Q_{0}(r)-\widetilde{Q}_{0}(r)\right|<\delta$ for $r \in \mathbb{R}^{+}$implies that $|Q(r, t)-\widetilde{Q}(r, t)| \leq \varepsilon$ for $0<r<N$ and $t>0$. 
According to the physical interpretation, $Q(r, t)$ should be a nondecreasing function of $r$. By the above remark it is enough to verify this fact for $Q$ starting from strictly increasing initial data $Q_{0}$.

THEOREM 3. If $Q_{0}^{\prime}(r)>0$ for each $r \geq 0$ then $Q_{r}(r, t)>0$ for $t \geq 0$, $r \geq 0$.

Proof. If $Q$ is solution of (5)-(7) then $W \equiv Q_{r}$ satisfies

$$
W_{t}=W_{r r}+Q W_{r}+W^{2} .
$$

Our assumptions and the Hopf lemma ([6]) applied to (7) imply the existence of a neighbourhood $U$ of the boundary of $\mathbb{R}_{+}^{2}$ such that $W(r, t)>0$ for $(r, t) \in U$. Assume that the set $B \equiv\left\{(r, t) \in \mathbb{R}_{+}^{2}: W(r, t)<0\right\}$ is not empty and let $\left(r_{0}, t_{0}\right)$ be a point of the boundary of $B$ nearest to the origin. Obviously $W\left(r_{0}, t_{0}\right)=0$. Applying the Hopf lemma in the domain $\{0 \leq r \leq$ $\left.r_{0}, 0 \leq t \leq t_{0}\right\}$ to $(19)$ we get $W_{r}\left(r_{0}, t_{0}\right) \neq 0$. Hence by the implicit function theorem there exists a unique function $r(t)$ defined on some neighbourhood $K$ of $t_{0}$ such that $W(r(t), t)=0$ and $r\left(t_{0}\right)=r_{0}$. Define the set $D \equiv\{(r, t)$ : $t \in K, r(t) \leq r \leq r(t)+\varepsilon\}$, where $\varepsilon$ is a small positive number so that $D \subset B$. The function $Q$ restricted to $D$ attains its maximum at some point $\left(r\left(t_{1}\right), t_{1}\right)$. By the Hopf lemma $Q_{r}\left(r\left(t_{1}\right), t_{1}\right) \neq 0$, which contradicts the definition of $r(t)$. In this way we proved that $Q_{r} \geq 0$. If $Q_{r}\left(r_{0}, t_{0}\right)=0$ then by the maximum principle applied to $(19), Q_{r}\left(r, t_{0}\right)=0$ for each $0 \leq r<+\infty$. This leads to a contradiction with the assumption (6).

Below we have an easy consequence of Remark 2 and Theorem 3.

Remark 3. The solution of the problem (5)-(7) depends continuously on the initial data.

2. In Section 1 we proved in an elementary way that for $M \geq 0$ and $n=1$ there exists a unique stationary solution of the problem (4)-(6). In higher dimensions the situation is more complicated. Note that for $n \geq 2$, with the help of a stationary solution $Q^{s}(r)$ we can produce a family of solutions putting $Q_{R}^{s}(r)=R^{2-n} Q^{s}(R r)$. Hence if a stationary solution exists, it is not unique (except for $Q^{s}(r)=c r^{n-2}$ ).

In the two-dimensional case it is convenient to introduce the variable $y=r^{2}$, which allows us to transform (4) into

$$
Q_{t}=4 y Q_{y y}+\pi^{-1} Q Q_{y} .
$$

Hence each stationary solution $Q^{s}$ of (20) satisfies the equation $4 \pi y Q_{y y}+$ $Q Q_{y}=0$, which is integrable, and its first integral is

$$
8 \pi y Q_{y}+Q(Q-8 \pi)=\text { const. }
$$


From (21) and the assumption $Q(0)=0$, after an integration we get

$$
Q^{s}(r)=8 \pi C r^{2} /\left(8 \pi-C+C r^{2}\right),
$$

where $C$ is a constant $0 \leq C<8 \pi$, which physically can be interpreted as the mass of particles in the ball of radius 1 . From (22) we see that $Q^{s}(+\infty)=8 \pi$. Hence in the two-dimensional case each nonzero stationary solution of (4)(6) has the total mass equal to $8 \pi$. In fact, it can be proved by applying the method of moving planes [5] that all stationary solutions of (3) in $\mathbb{R}^{2}$ with finite total mass are radially symmetric, hence have the form (22).

For the study of stationary solutions of (4) in $\mathbb{R}^{3}$ we use the phase plane method developed in [2], [8].

Assume that $Q^{s}$ is a stationary solution in $\mathbb{R}^{3}$. Introducing the new independent variable $\tau=\log r$ and putting

$$
v(r)=(4 \pi)^{-1} Q_{r}^{s}(r), \quad w(r)=(4 \pi r)^{-1} Q^{s}(r),
$$

we can check that $v$ and $w$ satisfy the system

$$
v^{\prime}=2 v-v w, \quad w^{\prime}=v-w, \quad{ }^{\prime}=\frac{d}{d \tau} .
$$

We are interested in the solutions of (24) which satisfy

$$
w(\tau) \geq 0, \quad v(\tau) \geq 0 .
$$

We prove that there are four trajectories of (24) satisfying (25): the line $\gamma_{1}=(v=0, w>0)$, the stationary points $P_{1}=(0,0), P_{2}=(2,2)$, and the separatrix $\gamma_{2}$ connecting $P_{1}$ to $P_{2}$.

Indeed, the origin is a saddle, $P_{2}$ is a sink and $L(v, w)=(w-2)^{2} / 2+$ $(v-2)-2 \log (v / 2)$ is a Lyapunov function for $(24)$. Hence the basin of attraction of $P_{2}$ contains the whole positive quadrant. Thus the separatrix $\gamma_{2}$ starting from $P_{1}$ ends in $P_{2}$, and turns around $P_{2}$ infinitely many times (the eigenvalues of the linearization of (24) at $P_{2}$ have imaginary parts equal to $\pm \sqrt{7})$.

Let us denote by $\beta_{1}, \beta_{2}$ two pieces of $\gamma_{2}$ :

$$
\begin{aligned}
\beta_{1}=\{(v(\tau), w(\tau)): & \tau \in\left(-\infty, \tau_{0}\right), \\
& \left.w(\tau) \leq 2 \text { for } \tau \in\left(-\infty, \tau_{0}\right), w\left(\tau_{0}\right)=2\right\}, \\
\beta_{2}=\{(v(\tau), w(\tau)): & \tau \in\left(\tau_{0}, \tau_{1}\right), w\left(\tau_{0}\right)=2, v\left(\tau_{1}\right)=2, \\
& \left.w(\tau) \neq 2 \text { for } \tau \in\left(\tau_{0}, \tau_{1}\right)\right\} .
\end{aligned}
$$

Obviously if the initial condition $(v(0), w(0))$ belongs to the domain bounded by the curve $\beta_{1}$ and the lines $(w=0, v>0), w=2$, then the solution of (24) passing through this point crosses the line $w=0$ at some moment $\tau<0$. It is easily seen that the solution $(v(\tau), w(\tau)) \neq P_{2}$ of $(24)$ starting from any point in the region bounded by $\gamma_{1}$, the line $\left(v=2, w \geq w\left(\tau_{1}\right)\right)$ and the curve $\beta_{1} \cup \beta_{2}$ crosses the line $(v=2, w>2)$ at some $\tau<0$. 
We claim that if $(v(\tau), w(\tau))$ is a solution of $(24)$ and $v(0)>2, w(0)>2$, then $v(\tau)=2$ for some $\tau<0$. Translating the origin to the point $(2,2)$ in the $(v, w)$ plane, and next introducing the polar coordinates $(r, \varphi),(24)$ becomes

$$
\begin{aligned}
\varphi^{\prime} & =\sin ^{2} \varphi+r \cos \varphi \sin ^{2} \varphi-\sin \varphi \cos \varphi+1 \equiv f_{1}(r, \varphi), \\
r^{\prime} & =-r \sin \varphi \cos \varphi-r^{2} \cos ^{2} \varphi \sin ^{2} \varphi-r \sin ^{2} \varphi \equiv f_{2}(r, \varphi) .
\end{aligned}
$$

Our claim follows from $f_{1}(r, \varphi)>0$ for $\varphi \in(0, \pi / 2)$. Hence the separatrix is the unique nonconstant solution $(v(\tau), w(\tau))$ of $(24)$ with $v(\tau)>0$ for all $\tau$.

Each trajectory $(v(\tau), w(\tau))$ of $(24)$ satisfying $(25)$ defines the stationary solution $Q^{s}(r)=4 \pi r w(r)$ of (4). Note that $Q_{r}^{s}=4 \pi v \geq 0$, hence $Q^{s}$ satisfies our physical assumption. $P_{1}$ corresponds to $Q^{s}(r) \equiv 0$. $P_{2}$ defines $Q^{s}(r)=8 \pi r$, the solution found by $\mathrm{S}$. Chandrasekhar in the thirties, in connection with his studies of the stellar structure.

The explicit form of the stationary solution corresponding to the separatrix $\gamma_{2}$ is not available. The separatrix $\gamma_{2}$ is a bounded curve which turns around $P_{2}$ infinitely many times. Hence for each $c \leq c_{0}=4 \pi \max w(\tau)$ there exists a stationary solution of $(24)$ such that $Q^{s}(1)=c$, and there is no stationary solution for $c>c_{0}$. Moreover, stationary solutions are unique for sufficiently small $c$, and there are infinitely many solutions for $c=8 \pi$; for $c \neq 8 \pi$ the number of solutions is finite but grows to infinity when $c$ approaches $8 \pi$.

$\gamma_{1}$ corresponds to the stationary solution $Q^{s}(r) \equiv$ const $>0$, which is not of physical interest.

Note that each stationary solution in $\mathbb{R}^{3}$ (except for $Q^{s}(r) \equiv$ const $\geq 0$ ) has infinite total mass and $Q^{s}(r) / r \rightarrow 8 \pi$ as $r \rightarrow+\infty$.

It is worth noting that the structure of the set of stationary solutions of the equation (4) considered in a bounded domain can be very complicated, and depends strongly on the geometry of this domain (cf. [8], [9]). Moreover, the explicit form of the solution is not available for $n \geq 3$.

In [2], [4] it was proved that, under some assumptions on initial data, solutions of (3) considered in a bounded domain blow up in finite time. We prove that a similar phenomenon occurs in $\mathbb{R}^{2}$.

THEOREM 4. If $Q(r, t)$ is a solution of $(4)-(6)$ with $n=2, Q_{0}(+\infty)>8 \pi$ and $Q_{r}(r, t)>0$, then $Q(r, t)$ ceases to exist after a finite time.

Proof. We will use equation (4) in the form (20). Choose $C$ such that $8 \pi<C<Q_{0}(+\infty)$. It follows from the implicit function theorem that there exists a smooth function $R(t)$ such that $Q(R(t), t)=C$. Define an auxiliary function

$$
W(t)=\int_{0}^{R(t)} Q(y, t) d y
$$


It is easy to check that

$$
\begin{aligned}
W^{\prime}(t) & =C R^{\prime}(t)+4 R(t) Q_{y}(R(t), t)-4 C+\frac{1}{2 \pi} C^{2} \\
& \geq C R^{\prime}(t)+\frac{1}{2 \pi} C^{2}-4 C .
\end{aligned}
$$

Integrating $(27)$ over $[0, t]$ we get

$$
W(t) \geq C R(t)-C R(0)+W(0)+\left(\frac{1}{2 \pi} C^{2}-4 C\right) t .
$$

From the obvious inequality $C R(t) \geq W(t)$ and $(28)$ we have $0 \geq W(0)-$ $C R(0)+\left(C^{2} /(2 \pi)-4 C\right) t$, which implies that the maximal time of the existence of solution does not exceed $(C R(0)-W(0)) /\left(C^{2} /(2 \pi)-4 C\right)$.

Remark 4. One can prove that the assumption $Q_{0}^{\prime}(r)>0$ in the twodimensional case also implies $Q_{r}(r, t)>0$ like for $n=1$. The arguments are similar to those used in the proof of Theorem 3. The only problem is that we cannot use directly the Hopf lemma, because the equation (20) is not uniformly parabolic. However, by inspection of the proof, we see that in our case the Hopf lemma is still valid. Hence we can use this argument in the proof of Remark 3 in the same manner as in Theorem 3.

Acknowledgements. The author would like to thank Piotr Biler and Andrzej Krzywicki for interesting discussions on related topics.

\section{References}

[1] P. Biler, The Cauchy problem and self-similar solutions for a nonlinear parabolic equation, preprint 1994.

[2] P. Biler, D. Hilhorst and T. Nadzieja, Existence and nonexistence of solutions for a model of gravitational interaction of particles, II, Colloq. Math. 67 (1994), 297-308.

[3] P. Biler and T. Nadzieja, A class of nonlocal parabolic problems occurring in statistical mechanics, ibid. 66 (1993), 131-145.

[4] - - - Existence and nonexistence of solutions for a model of gravitational interaction of particles, I, ibid. 66 (1994), 319-334.

[5] W. Chen and C. Li, Classification of solutions of some nonlinear elliptic equations, Duke Math. J. 63 (1991), 615-622.

[6] A. Friedman, Partial Differential Equations of Parabolic Type, Prentice-Hall, Englewood Cliffs, N.J., 1964.

[7] E. Hopf, The partial differential equation $u_{t}+u u_{x}=u_{x x}$, Comm. Pure Appl. Math. 3 (1950), 201-230.

[8] A. Krzywicki and T. Nadzieja, Some results concerning the Poisson-Boltzmann equation, Zastos. Mat. 21 (1991), 265-272.

[9] - - - A note on the Poisson-Boltzmann equation, ibid. 21 (1993), 591-595. 
[10] G. Wolansky, On steady distributions of self-attracting clusters under friction and fluctuations, Arch. Rational Mech. Anal. 119 (1992), 355-391.

TADEUSZ NADZIEJA

MATHEMATICAL INSTITUTE

UNIVERSITY OF WROCłAW

PL. GRUNWALDZKI $2 / 4$

50-384 WROCłAW, POLAND

E-mail: NADZIEJA@MATH.UNI.WROC.PL

Received on 25.7.1994 